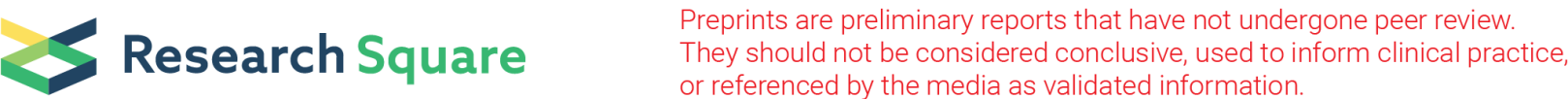

\section{ACE2 Expression in Lungs of Severe COVID-19 Infection: A Study on Minimally Invasive Post- mortem Tissue Samples}

\section{Atish Gheware}

All India Institute of Medical Sciences (AlIMS)

\section{Animesh Ray}

All India Institute of Medical Sciences (AlIMS)

\section{Deeksha Rana}

All India Institute of Medical Sciences (AIIMS)

\section{Prashant Bajpai}

International Centre for Genetic Engineering and Biotechnology

\section{Aruna Nambirajan}

All India Institute of Medical Sciences (AlIMS)

\section{S Arulselvi}

All India Institute of Medical Sciences (AlIMS)

\section{Purva Mathur}

All India Institute of Medical Sciences (AIIMS)

\section{Anjan Trikha}

All India Institute of Medical Sciences (AIIMS)

\section{Sudheer Arava}

All India Institute of Medical Sciences (AlIMS)

\section{Prasenjit Das}

All India Institute of Medical Sciences (AlIMS)

\section{Asit Ranjan Mridha}

All India Institute of Medical Sciences (AlIMS)

\section{Geetika Singh}

All India Institute of Medical Sciences (AIIMS)

\section{Manish Soneja}

All India Institute of Medical Sciences (AIIMS)

\section{Neeraj Nischal}

All India Institute of Medical Sciences (AlIMS)

\section{Sanjeev Lalwani}

All India Institute of Medical Sciences (AlIMS) 
All India Institute of Medical Sciences (AlIMS)

\section{Chitra Sarkar}

All India Institute of Medical Sciences (AlIMS)

Deepali Jain ( $\sim$ deepalijain76@gmail.com )

All India Institute of Medical Sciences (AlIMS)

\section{Research Article}

Keywords: ACE2, COVID-19 histopathology, SARS-CoV-2, macrophage, coagulopathy

Posted Date: August 19th, 2021

DOI: https://doi.org/10.21203/rs.3.rs-805896/v1

License: (9) This work is licensed under a Creative Commons Attribution 4.0 International License. Read Full License 


\section{Abstract}

Angiotensin-converting enzyme 2 (ACE2) is a key host protein by which severe acute respiratory syndrome coronavirus-2 (SARS-CoV-2) enters and multiplies within cells. The level of ACE2 expression in the lung is hypothesised to correlate with an increased risk of severe infection and complications in COVID-19 (COrona VIrus Disease 2019). To test this hypothesis, we compared the protein expression status of ACE2 by immunohistochemistry (IHC) in post-mortem lung samples of patients who died of severe COVID-19 and lung samples obtained from non-COVID-9 patients for other indications. IHC for CD61 and CD163 were performed for assessment of platelet-rich microthrombi and macrophages, respectively. IHC for SARS-CoV-2 viral antigen was also performed. Quantification of immunostaining, random sampling, and correlation analysis was used to substantiate the morphologic findings. Our results show that among a total of 44 COVID-19 post-mortem lung tissues and 15 lung biopsies in nonCOVID-19 patients included, ACE2 protein expression was significantly higher in COVID-19 patients than in controls, regardless of sample size. Histomorphology in COVID-19 lungs showed diffuse alveolar damage (DAD), acute bronchopneumonia, and acute lung injury with SARS-CoV-2 viral protein detected in a subset of cases. ACE2 expression levels positively correlated with increased expression levels of CD61 and CD163. In conclusion, our results show significantly higher ACE2 protein expression in severe COVID19 disease, correlating with increased macrophage infiltration and microthrombi, suggesting a pathobiological role in disease severity.

\section{Introduction}

The pneumonia epidemic COVID-19 (COrona VIrus Disease 2019) triggered by severe acute respiratory syndrome coronavirus- 2 (SARS-CoV-2) is spreading at an alarming rate ${ }^{1,2}$. The membrane-bound angiotensin-converting enzyme-2 (ACE-2), which is expressed in various human organs, has been identified as the SARS-CoV-2 entry receptor ${ }^{3,4}$. ACE2 is a negative regulator of the renin-angiotensin pathway. It inhibits the effects of angiotensin-II (Ang II) and its structurally related receptor ACE, thereby negatively regulating vasoconstriction, cell proliferation, and inflammation ${ }^{5,6}$. The role of ACE2 expression in COVID-19 disease pathogenesis is not well understood, and whether the level of ACE2 expression levels (mRNA or protein) relates with the risk of infection or disease severity is not known. At the transcriptional level, ACE2 mRNA is normally detected at low levels in the respiratory system ${ }^{6,7}$. In COVID-19 disease, studies have shown higher ACE2 mRNA levels in patients' nasal swabs, bronchial brushes, and bronchoalveolar lavages ${ }^{8}$ although more recent in-vitro studies did not find any such ACE2 mRNA elevation ${ }^{9,10}$. Immunohistochemistry (IHC) analysis of lungs from a small number of COVID-19 patients revealed a higher number of alveolar epithelial cells expressing ACE2 protein ${ }^{11,12}$. This is in contrast to a cell culture study that demonstrated decreased ACE2 protein levels after 24 hours of infection in SARS-CoV-2 transfected cells ${ }^{13}$. Thus, there is no clear data on ACE2 mRNA or protein expression levels during SARS-CoV-2 infection. In particular, there is an incredible scarcity of studies evaluating ACE2 protein expression in COVID-19 patient lung samples. Given the current scenario where individual risk factors for severe COVID-19 disease still remain unclear, this cross-sectional observational 
study aimed to assess ACE2 protein expression in the lung parenchyma of fatal COVID-19 cases in correlation with histopathology, markers of vascular and inflammatory responses, and clinical features to ascertain its role, if any, in disease severity and potential for therapeutic targeting.

\section{Results}

\section{Patients' clinical data and pathological finding}

A total of 55 lung samples obtained from deceased COVID-19 patients were included. Patients included 38 men and 17 women, with a median age of 49 years (SD 17.27; range 13-82) at death. All patients tested positive for SARS-CoV-2 using nasopharyngeal swab PCR. Shortness of breath (49\%), fever (40\%), and cough $(21.8 \%)$ were the most common symptoms at onset. Status regarding time spent in the hospital was available for 36 patients, and the mean length of stay of these patients in the critical care unit or intermediate medical ward was 12.6 days. Respiratory failure or multiorgan failure involving the respiratory system was the leading causes of death. In terms of previous comorbidities, $11(20 \%)$ had diabetes, 15 (27.27\%) had cardiovascular complications, 9 (16.3\%) had previous respiratory illnesses, 18 $(32.7 \%)$ had liver or renal diseases, and five (9\%) had cancer. Additionally, 22 patients had other complications such as hypothyroidism, anaemia, steroid-induced hypoglycaemia, or dyslipidaemia (Table 1 and supplemental figure $\mathrm{S} 1$ ).

Table 1

Patient clinicopathological information 


\begin{tabular}{|c|c|c|}
\hline Characteristics & Nor mean & $\%$ or rang \\
\hline \multicolumn{3}{|l|}{ COVID-19 cohort (55 patients) } \\
\hline Age & $46 y$ & $13-82 y$ \\
\hline Sex & M 38, F17 (2.2:1) & \\
\hline Hospitalisation time & $12.6 \mathrm{~d}$ & $1-88 d$ \\
\hline \multicolumn{3}{|l|}{ Major symptoms on admission } \\
\hline Fever & 22 & $40.00 \%$ \\
\hline Cough & 12 & $21.82 \%$ \\
\hline Shortness of breath & 27 & $49.09 \%$ \\
\hline \multicolumn{3}{|l|}{ Major comorbidities } \\
\hline Diabetes & 11 & $20.00 \%$ \\
\hline Cardiovascular complications & 15 & $27.27 \%$ \\
\hline Respiratory complications & 9 & $16.36 \%$ \\
\hline Cancer & 5 & $9.09 \%$ \\
\hline Liver or kidney complications & 18 & $32.73 \%$ \\
\hline Thyroid complications & 5 & $9.09 \%$ \\
\hline Aplastic anaemia & 2 & $3.64 \%$ \\
\hline Patients with no prior comorbidities & 4 & $7.27 \%$ \\
\hline \multicolumn{3}{|l|}{ Most common lung pathological features } \\
\hline Exudative phase of diffuse alveolar damage & 25 & $45.45 \%$ \\
\hline Organizing phase of diffuse alveolar damage & 13 & $23.64 \%$ \\
\hline Acute bronchopneumonia & 5 & $9.09 \%$ \\
\hline Acute lung injury & 9 & $16.36 \%$ \\
\hline No changes & 3 & $5.45 \%$ \\
\hline Coexisting acute bronchopneumonia with DAD & 12 & \\
\hline \multicolumn{3}{|l|}{ Control cohort (15 subjects) } \\
\hline Age & $43.1 y$ & 18-70y \\
\hline Sex & M 8, F $7(1: 1)$ & \\
\hline
\end{tabular}




\begin{tabular}{lll} 
Normal lung & 13 & $86.66 \%$ \\
\hline Diffuse alveolar haemorrhage & 1 & 6.66 \\
\hline Diffuse alveolar damage & 1 & $7 \%$
\end{tabular}

On histopathology, majority of cases demonstrated histological evidence of exudative and organising phases of diffuse alveolar damage (69\%), acute bronchopneumonia (9\%), and acute lung injury (16.3\%) (Figure 1 and Table 1, and supplementary figure S1). Additionally, two individuals had bacterial microabscesses assumed to have developed during hospitalisation. Minority cases (5.4\%) did not show any significant histomorphological changes (Table 1).

\section{SARS-CoV-2 expression}

A subset of lung sections $(n=10)$ from COVID-19 patients showed presence of SARS-CoV2 viral antigen using IHC (Figure 1B). Among the ten subjects, eight have diffuse alveolar damage on histopathology (Supplementary Table S1).

\section{Control patients' clinical data and histopathological findings}

A total of 15 lung sections from control patients were included for comparison. The median age of this cohort was 48 years with male: female ratio of 1:1 (Table 1). All control patients had tested negative for SARS-CoV-2 using nasopharyngeal swab PCR. The control cohort's

characteristics are summarised in Table 1 and Supplementary Table S1. Histopathology was normal in all control patients, except for two patients whose lung biopsy showed evidence of diffuse alveolar haemorrhage and diffuse alveolar damage (Table 1 and Supplementary Table S2). Two sections, each, of normal kidneys, testes, and adrenal glands were also stained for ACE2 (Figure 2A-C).

\section{ACE2 protein expression in cases and controls}

ACE2 immunohistochemistry was performed in 44 cases and 15 controls (Supplementary Figure S1 and Table S1). ACE2 protein was largely undetectable $(n=6)$ or was detected exclusively in the cytoplasm of rare type-Il pneumocytes $(n=7)$ in the histologically normal control lungs (Supplementary Table S1). Expression was observed at multiple foci in the two control biopsies that showed diffuse alveolar haemorrhage (DAH) and diffuse alveolar damage (DAD) (Supplementary Table S1). The mean intensity for ACE2 protein was $2.8 \pm 0.63$ while was 14.5 and 4.9 in the two cases with DAD and DAH respectively.

ACE2 protein expression was higher in sections from the normal kidney, testes and adrenal glands (figure $2 A-C)$. Additionally, quantitative imaging analysis revealed that the mean intensity of ACE2 protein is substantially lower in control lung $(2.8 \pm 0.63)$ sections than in control kidney $(19.5 \pm 2.57)$, testis (66.3 \pm 7.38$)$, and adrenal (12.1 \pm 1.52$)$ tissues (Figure $2 \mathrm{~F}$ ). 
In lung tissues from COVID-19 patients, the mean ACE2 expression was $7 \pm 0.56$, with $86 \%$ cases showing higher mean intensity than the control lung samples (2.8 \pm 0.63 ) (Figure $2 \mathrm{D}, 2 \mathrm{E}$ and $2 \mathrm{G}$ ), including one sample with relatively normal histomorphology (3.12 mean intensity). This difference reached strong statistical significance $(P=<0.0001)$ (Figure $2 \mathrm{G}$ ). Random sampling analysis further confirmed the significant increase in ACE2 protein in the lungs of COVID-19 patients (Figure 2H), negating any bias in the number of samples and images in the control group.

\section{CD163 and CD61 expression levels}

CD163 and CD61 staining were used to examine the inflammatory and coagulopathy components of COVID-19. CD163 and CD61 immunohistochemistry was performed in 36 and 26 cases (Figure S1) and 6 control samples each of normal lungs, respectively. Among control tissue and cases, macrophagespecific inflammation and platelet plugs and/or platelet-rich microthrombi were found exclusively in COVID-19 lung sections (Figure 3A-F). Quantitative IHC analysis demonstrated that patients with COVID19 had significantly higher mean intensities of CD163 ( $8.5 \pm 2.20$ vs. $26.3 \pm 1.15, P=<0.0001)$ and CD61 $(0.28 \pm 0.04$ vs. $10 \pm 0.78, P=<0.0001)$ staining than those without (Figure $3 \mathrm{C}$ and $3 \mathrm{~F}$ ). Even after random sampling analysis, CD163 and CD61 protein expression remained significantly higher in COVID-19 patients (Figure $3 \mathrm{G}$ and $3 \mathrm{H}$ ). Linear regression analysis revealed a significant association between ACE2 expression and coagulation and inflammatory marker levels in paired samples from cases and controls ( $n=26$ for $\mathrm{CD} 163$ and $n=16$ for CD61) (Figure $3 \mathrm{I}$ and $3 \mathrm{~J}$ ).

\section{Discussion}

It is well established that the ACE2 protein acts as the entry receptor for the SARS-CoV-2 and the closely related SARS-CoV virus. ACE2 protein expression varies in different organs and its expression level appears to correlate with vulnerability of individual organs to infection. In the respiratory tract, ACE2 gene expression levels have been found to be highest in the olfactory bulbs, lower in the nasal respiratory epithelium and least in the bronchioles and alveoli $6,7,14$. At the protein level, however, ACE2 expression is weakly detectable in the lungs and shows poor correlation with mRNA levels ${ }^{11-13}$. By immunohistochemistry, the present study demonstrated negligible expression of ACE2 protein in normal lung parenchyma ${ }^{11,12}$. The expression was seen mainly in scattered Type 2 pneumocytes and compared to bulk analyses of entire tissues of other organs, its expression is less ${ }^{6,7,14}$. We found higher ACE2 protein levels in kidneys (proximal tubular cells), testis and adrenal glands (Fig. 2A-C and 2F), the latter two added to the list of organs that may be infected during viremia. Although

not analysed in the present study, cardiac myocytes, endothelial cells, bile duct epithelial cells, and the intestinal mucosal cells are other normal tissues that are known to express ACE2 at high levels and are vulnerable to SARS-CoV-2 infection. Notably, COVID-19 disease presenting with intestinal symptoms or cardiac events is not uncommon. Age and gender-based variations in the amounts of membrane-bound and circulating ACE2 have been reported with levels being high in men as compared to women and least 
in children ${ }^{15-17}$. However, in our small control cohort of mainly adults, we did not find any significant difference in ACE2 protein expression based on gender.

Despite low levels of baseline ACE2 protein expression, the lung parenchyma represents one of the major organs involved in severe COVID-19 disease $8,18,19$. The classical RAS activation ACE-Ang II-AT1R axis is known to exacerbate lung damage and inflammation and a shift toward the ACE2/angiotensin-(17)/MAS RAS axis is hypothesized to act as a protective counteractive mechanism ${ }^{5,6,20,21}$. It is also postulated that SARS-CoV-2 infection may decrease the availability of ACE2 for AT1 and ACE degradation, resulting in Angll-dependent lung damage and edema and in increased ACE2 levels 5,6,20,21. In our cohort of patients with fatal COVID-19 disease, we observed significantly increased ACE2 protein expression levels in the lung parenchyma as compared to control lung tissues (Fig. 2). Notably, all of these tissues showed histological evidence of severe lung parenchymal injury manifested as diffuse alveolar damage (exudative and early proliferative phases) with or without bronchopneumonia and/or microthrombi with no meaningful link between histological severity and ACE intensity, particularly in low and moderate ACE staining cases (Supplementary Figure S1). SARS-CoV-2 viral protein was demonstrated in a subset of cases and a linear positive correlation was observed between ACE2 and CD61/CD163 expression levels representing the inflammatory cascade and vasculopathy in COVID-19 disease respectively (Fig. $3 \mathrm{I}$ and $3 \mathrm{~J}$ ). All the above findings suggest that ACE2 expression levels are upregulated in the course of severe COVID-19 pulmonary disease. However, whether this ACE2 upregulation directly contributes to disease severity or is a compensatory protective mechanism or just a bystander phenomenon unrelated to disease severity remains unanswered. A comparison among lung tissue from SARS-CoV-2 infected patients with asymptomatic, mild and severe COVID-19 disease would be ideal for understanding these mechanisms but is beyond the scope of this study.

Given that we found negligible expression of ACE2 in non-COVID-19 lungs irrespective of gender, and uniform increase in ACE2 expression in all severe COVID-19 lungs, it seems unlikely that baseline pulmonary ACE2 expression levels contribute to the risk of developing COVID-19 pulmonary disease in infected patients. However, expression, correlation, and network analyses have revealed increased levels of ACE2 in the lungs of individuals with respiratory illnesses (COPD, acute respiratory distress syndrome), cardiovascular disease, hypertension, diabetes, chronic renal disease, and cancer 5,6,17,22-26 and it is well known that individuals with such co-morbidities are susceptible to severe COVID-19 disease, likely due to increased ACE2 expression allowing for greater viral invasiveness. Although our control cohort did not include many patients with such co-morbidities, we did notice increased ACE2 expression in two nonCOVID19 patients with diffuse alveolar damage and alveolar haemorrhage supporting that the higher ACE2 expression in diseased lungs may contribute to the increased disease susceptibility and severity $22,26-28$.

To conclude, the present study performed on a relatively large cohort of COVID-19 lung samples demonstrates high pulmonary expression of ACE2 protein in severe and fatal COVID-19 disease and 
negligible expression in control lung tissue, highlighting an important role for ACE2 protein in the pathogenesis of SARS-CoV-2 infection.

\section{Methods}

\section{Patient sample and tissue processing}

The study has been approved by the Institute Ethics Committee of the All India Institute of Medical Sciences at New Delhi, India (IEC-536/05.06.2020, OP-05/03.07.2020). All methods in this study were performed in accordance with the guidelines and regulations by the Indian Council of Medical Research (ICMR), Institute human ethics committee, and hospital for conducting scientific research. Cases comprised of post-mortem lung samples obtained from patients who died due to severe COVID-19 infection during the study period after providing informed written consent from next of kin. Control tissues were obtained from subjects who underwent lung biopsies, surgical resections, or autopsies for other causes during the study period. For all control subjects, informed consent was obtained, either personal and/or immediate patient representative for sample collection. Normal kidney, testis, and adrenal tissue were also obtained from the surgical pathology laboratory for comparison of ACE2 expression in different tissue samples. The samples were fixed in formalin and processed into formalinfixed paraffin-embedded (FFPE) blocks. Blocks were cut into 4- $\mu \mathrm{m}$ sections and stained with haematoxylin and eosin using a standardised procedure.

\section{Histopathological evaluation}

Histopathological evaluations were performed on lung sections stained with haematoxylin and eosin. Two thoracic pathologists (DJ and AN) independently evaluated the slides. The following features: extent of lung damage, injury, inflammation, presence or absence of hyaline membrane formation, lymphocyte infiltration, organising pneumonia, alveolar fibrin deposition, fibrosis, and histologic features of type 2 pneumocyte hyperplasia, were noted and documented.

\section{Immunohistochemistry}

Tissue blocks were cut into 4- $\mu \mathrm{m}$ sections, cleaned in xylene and hydrated through alcohol series to distilled water. After antigen retrieval, endogenous peroxidase activity was blocked by treating the sections with $4 \%$ hydrogen peroxide for $30 \mathrm{~min}$. Slides were washed with buffer (TRIS Buffer) and blocked with background Protein Block (Mouse and rabbit Specific HRP (ABC) Detection IHC kit ab93677, abcam) for five min and washed with buffer. The area around the tissue was marked with PAP pen. Tissue sections were then incubated overnight with ACE-2 antibody (1:400, ACE-2 Recombinant Rabbit Monoclonal Antibody, SN0754, Invitrogen, USA), CD-61 antibody (1:200, CD61 BIO SB Clone-Ep65 RMab, Cell Signaling Technology), CD-163 antibody (1:200, CD163, Monoclonal Antibody (10D6) Invitrogen) and SARS CoV2 antibody (1:100, SARS, Coronavirus Nucleocapsid Monoclonal Antibody (B46F) Invitrogen). 
After washing, the tissue section was processed with the ABC Detection kit (Mouse and rabbit Specific HRP (ABC) Detection IHC kit ab93677, abcam), according to the manufacturer's protocol, and HRP activity was detected using the DAB (SignalStrain (R) DAB substrate kit, Cell Signaling Technology). Finally, the sections were counterstained with hematoxylin. Presence of ACE2, CD163, and CD61 staining of any intensity in the cytoplasm +/- membranous area was defined as positive staining.

\section{Quantification of ACE2, CD61, and CD163 in lung parenchyma}

The staining was examined using an Olympus bright-field microscope at magnifications of 100x and 200x. CellSens imaging software (Olympus, India) was used to photograph the slides. Stains were assessed by imaging the most highly stained portions at least twice across distinct locations, ensuring not to sample the same location more than once. The images captured above at $100 \times$ magnification for CD163 and 200x magnification for ACE2 and CD61, were then quantified using ImageJ software (National Institutes of Health). H-DAB colour-based deconvolution tool was used for the separation of DAB staining images. Adjustments were made to the thresholds in order to produce sharp particles and eliminate background noise. The generated picture was then processed to determine the total positive area expressed in terms of mean intensity. The intensity value of each image from tissue section was considered separately for analysis rather than averaging intensities across all images. This was done to avoid any biologically meaningful normalisation of intensities across images of a tissue section. For grading low, medium, and high intensity the mean IHC expression values per sample were divided into quartiles. For each group, values up to the 25th quartile was classified as 'low', those between the 25th and 75th quartiles as 'medium,' and those between the 75th and 100th quartiles as 'high.'

\section{Assessment of SARS-CoV-2 expression in lung parenchyma}

For SARS CoV2 antibody, cytoplasmic staining of any intensity was taken as positive expression as described previously 29

\section{Statistical Analysis}

Difference between the means of the sample groups was calculated using Wilcoxon test wherever the data was not normally distributed. Pearson correlation coefficient was used for estimating correlations and regression line was drawn using linear fit. Random sampling was performed to account for imbalance in number of samples in each sample group. For example, ACE2 samples had 45 intensities values in Control and 180 in COVID-19. To minimize the bias arising due to differences in sample numbers, same number of intensities values were randomly sampled from COVID-19 group. The same methodology was employed for CD163 and CD61 samples. While calculating the correlations, only the 
samples which had paired data for the groups being compared were selected. Statistical analysis was performed with R 4.0.3 software. $P<0.05$ was considered to be statistically significant.

\section{Declarations}

\section{Acknowledgment}

We would like to express our gratitude to all healthcare personnel who are fighting SARSCOV-2 on the front lines. We would like to express our sincere thanks to Prashant Chowdhary for giving technical assistance with immunohistochemistry.

\section{Funding:}

This work is supported by intramural funding from the research section of the All India Institute of Medical Sciences (AlIMS), New Delhi, India (Grant Number A-COVID 40).

\section{Declarations of interest:}

All authors declare no conflicts of interest.

\section{Author contributions}

DJ conceptualised the study, design the experiments, interpreted histopathology of samples, analysed and interpreted the study results, and wrote the manuscript. AG designed the article, analysed and interpreted the results, and wrote the manuscript. DR performed the experiments and analysed the results. PB contributed to data analysis and representation. AN interpreted histopathology of samples, analysed the results, designed, and wrote the manuscript. AR, SA, PM, AT, NW, MS, NN, and SL contributed to acquisition of samples and data collection. SA, PD, ARM, GS, and CS contributed to the interpretation of histopathology of specimens. All authors read and approved the final manuscript.

\section{References}

1. Zhu, N. et al. A Novel Coronavirus from Patients with Pneumonia in China, 2019. N. Engl. J. Med, 382, 727-733 (2020).

2. Zou, L. et al. SARS-CoV-2 Viral Load in Upper Respiratory Specimens of Infected Patients. N. Engl. J. Med, 382, 1177-1179 (2020).

3. Wan, Y., Shang, J., Graham, R., Baric, R. S. \& Li, F. Receptor Recognition by the Novel Coronavirus from Wuhan: an Analysis Based on Decade-Long Structural Studies of SARS Coronavirus.J. Virol.94, (2020). 
4. Hoffmann, M. et al. SARS-CoV-2 Cell Entry Depends on ACE2 and TMPRSS2 and Is Blocked by a Clinically Proven Protease Inhibitor., 181, 271-2808 (2020).

5. Medina-Enríquez, M. M. et al. ACE2: the molecular doorway to SARS-CoV-2. Cell Biosci, 10, 148 (2020).

6. Zamorano Cuervo, N. \& Grandvaux, N. ACE2: Evidence of role as entry receptor for SARS-CoV-2 and implications in comorbidities. Elife9, (2020).

7. Ortiz, M. E. et al. Heterogeneous expression of the SARS-Coronavirus-2 receptor ACE2 in the human respiratory tract. EBioMedicine, 60, 102976 (2020).

8. Chua, R. L. et al. COVID-19 severity correlates with airway epithelium-immune cell interactions identified by single-cell analysis. Nat. Biotechnol, 38, 970-979 (2020).

9. Onabajo, O. O. et al. Interferons and viruses induce a novel truncated ACE2 isoform and not the fulllength SARS-CoV-2 receptor. Nat. Genet, 52, 1283-1293 (2020).

10. Blume, C. et al. A novel ACE2 isoform is expressed in human respiratory epithelia and is upregulated in response to interferons and RNA respiratory virus infection. Nat. Genet, 53, 205-214 (2021).

11. Ackermann, M. et al. Pulmonary vascular endothelialitis, thrombosis, and angiogenesis in Covid-19. N. Engl. J. Med, 383, 120-128 (2020).

12. Damiani, S. et al. Pathological post-mortem findings in lungs infected with SARS-CoV-2. J. Pathol, 253, 31-40 (2021).

13. Blanco-Melo, D. et al. Imbalanced Host Response to SARS-CoV-2 Drives Development of COVID-19., $181,1036-10459$ (2020).

14. Hou, Y. J. et al. SARS-CoV-2 Reverse Genetics Reveals a Variable Infection Gradient in the Respiratory Tract., 182, 429-44614 (2020).

15. Swärd, P. et al. Age and sex differences in soluble ACE2 may give insights for COVID-19. Crit. Care, 24, 221 (2020).

16. Ciaglia, E., Vecchione, C. \& Puca, A. A. COVID-19 Infection and Circulating ACE2 Levels: Protective Role in Women and Children. Front. Pediatr, 8, 206 (2020).

17. Pathangey, G., Fadadu, P. P., Hospodar, A. R. \& Abbas, A. E. Angiotensin-converting enzyme 2 and COVID-19: patients, comorbidities, and therapies. Am. J. Physiol. Cell. Mol. Physiol, 320, L301-L330 (2021).

18. Bar-On, Y. M., Flamholz, A., Phillips, R. \& Milo, R. Sars-cov-2 (Covid-19) by the numbers.Elife9, (2020).

19. Nienhold, R. et al. Two distinct immunopathological profiles in autopsy lungs of COVID-19. Nat. Commun, 11, 5086 (2020).

20. Heyman, S. N., Kinaneh, S. \& Abassi, Z. The Duplicitous Nature of ACE2 in COVID-19 Disease. EBioMedicine, 67, 103356 (2021).

21. South, A. M., Tomlinson, L., Edmonston, D., Hiremath, S. \& Sparks, M. A. Controversies of reninangiotensin system inhibition during the COVID-19 pandemic. Nat. Rev. Nephrol, 16, 305-307 (2020). 
22. Pinto, B. G. G. et al. ACE2 Expression Is Increased in the Lungs of Patients With Comorbidities Associated With Severe COVID-19. J. Infect. Dis, 222, 556-563 (2020).

23. Wijnant, S. R. A. et al. Expression of ACE2, the SARS-CoV-2 Receptor, in Lung Tissue of Patients With Type 2 Diabetes., 69, 2691-2699 (2020).

24. Jacobs, M. et al. Increased expression of ACE2, the SARS-CoV-2 entry receptor, in alveolar and bronchial epithelium of smokers and COPD subjects.Eur. Respir. J.56, (2020).

25. Dai, Y. J. et al. A profiling analysis on the receptor ACE2 expression reveals the potential risk of different type of cancers vulnerable to SARS-CoV-2 infection. Ann. Transl. Med, 8, 481-481 (2020).

26. Zhou, F. et al. Clinical course and risk factors for mortality of adult inpatients with COVID-19 in Wuhan, China: a retrospective cohort study., 395, 1054-1062 (2020).

27. Shi, S. et al. Characteristics and clinical significance of myocardial injury in patients with severe coronavirus disease 2019. Eur. Heart J, 41, 2070-2079 (2020).

28. Marietta, M. et al. COVID-19 and haemostasis: A position paper from Italian Society on Thrombosis and Haemostasis (SISET). Blood Transfus, 18, 167-169 (2020).

29. Ko, C. J. et al. Discordant anti-SARS-CoV -2 spike protein and RNA staining in cutaneous perniotic lesions suggests endothelial deposition of cleaved spike protein. J. Cutan. Pathol, 48, 47-52 (2021).

\section{Figures}


A

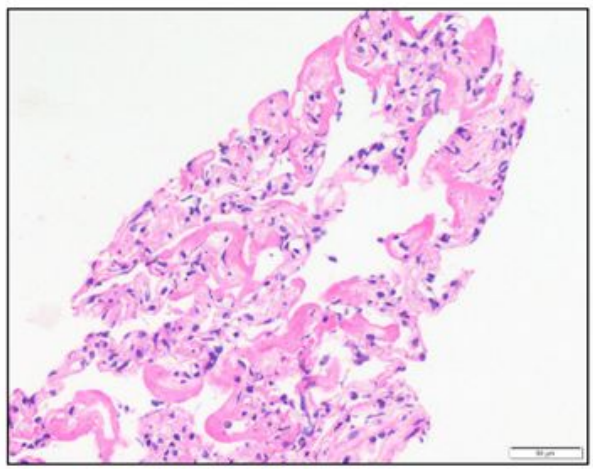

C

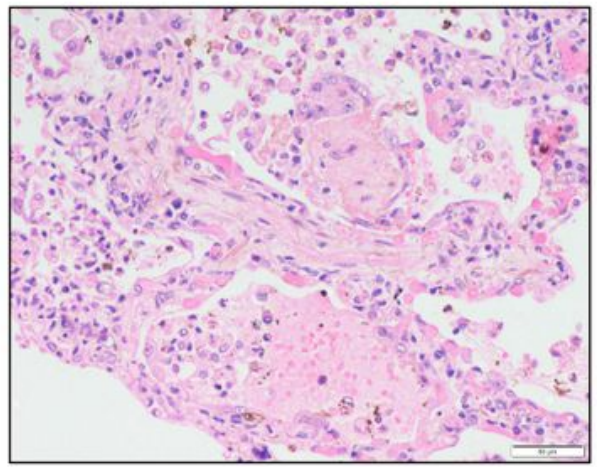

E

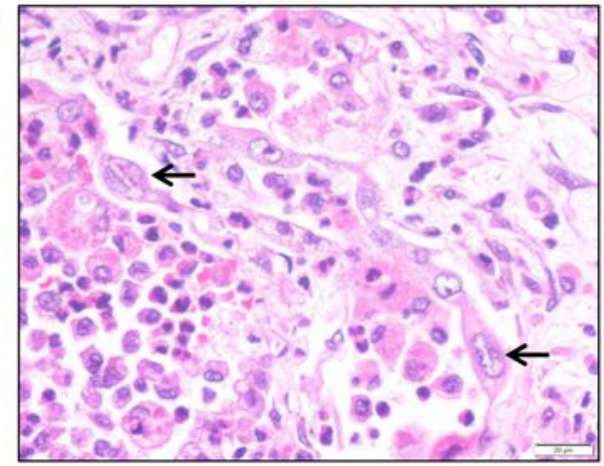

B

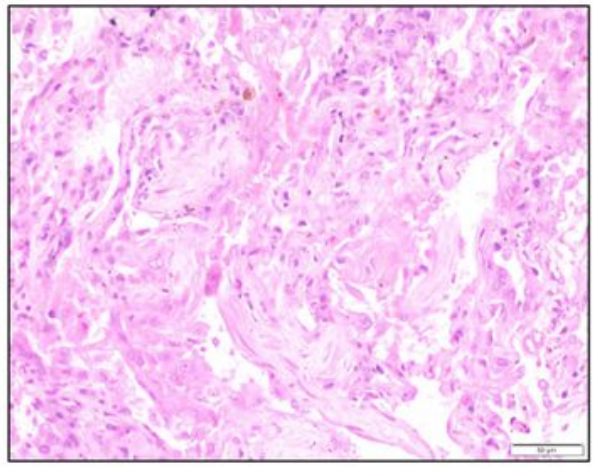

D

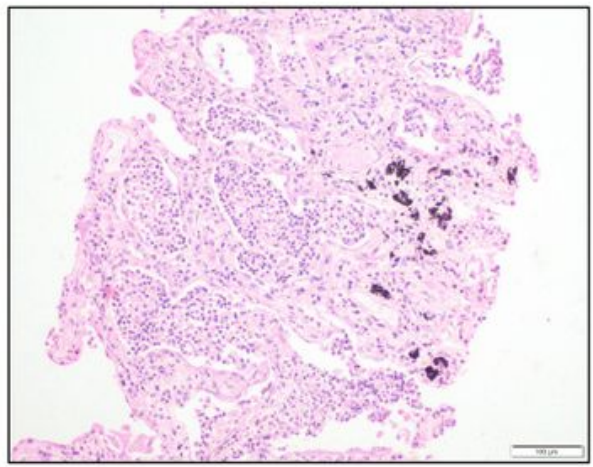

F

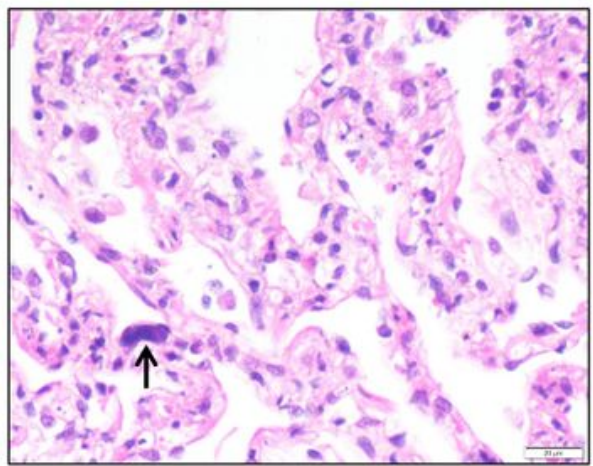

G

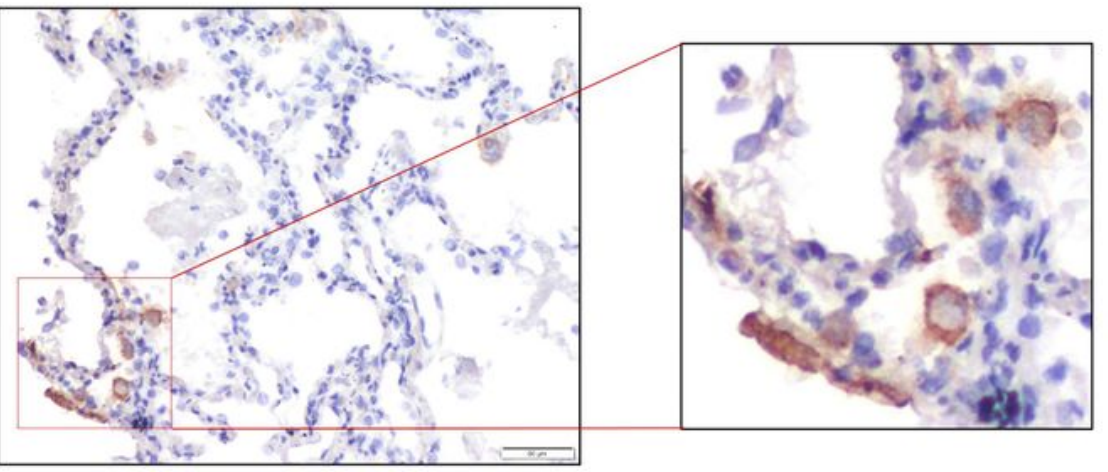

\section{Figure 1}

Haematoxylin and eosin-stained sections from representative areas of lung parenchyma infected with COVID-19. The microphotograph shows (A) diffuse alveolar damage with the hyaline membrane (exudative phase) (x200). (B) Diffuse alveolar damage with organization (organizing phase) (x 200). (C) Organizing pneumonia with interalveolar capillaries filled with microthrombi (x200). (D) Alveolar spaces are filled with neutrophilic infiltrate in a case of organizing DAD (not shown here) indicate superimposed 
acute bronchopneumonia (x100). (E and F) Hyperplastic pneumocytes (E, arrow) and megakaryocytes (F, arrow) are seen in a case of DAD with acute bronchopneumonia (x400). (G) Representative image of SARS-CoV2-stained tissue from COVID-19 patient. SARS-COV2 IHC shows cytoplasmic granular positivity in pneumocytes.
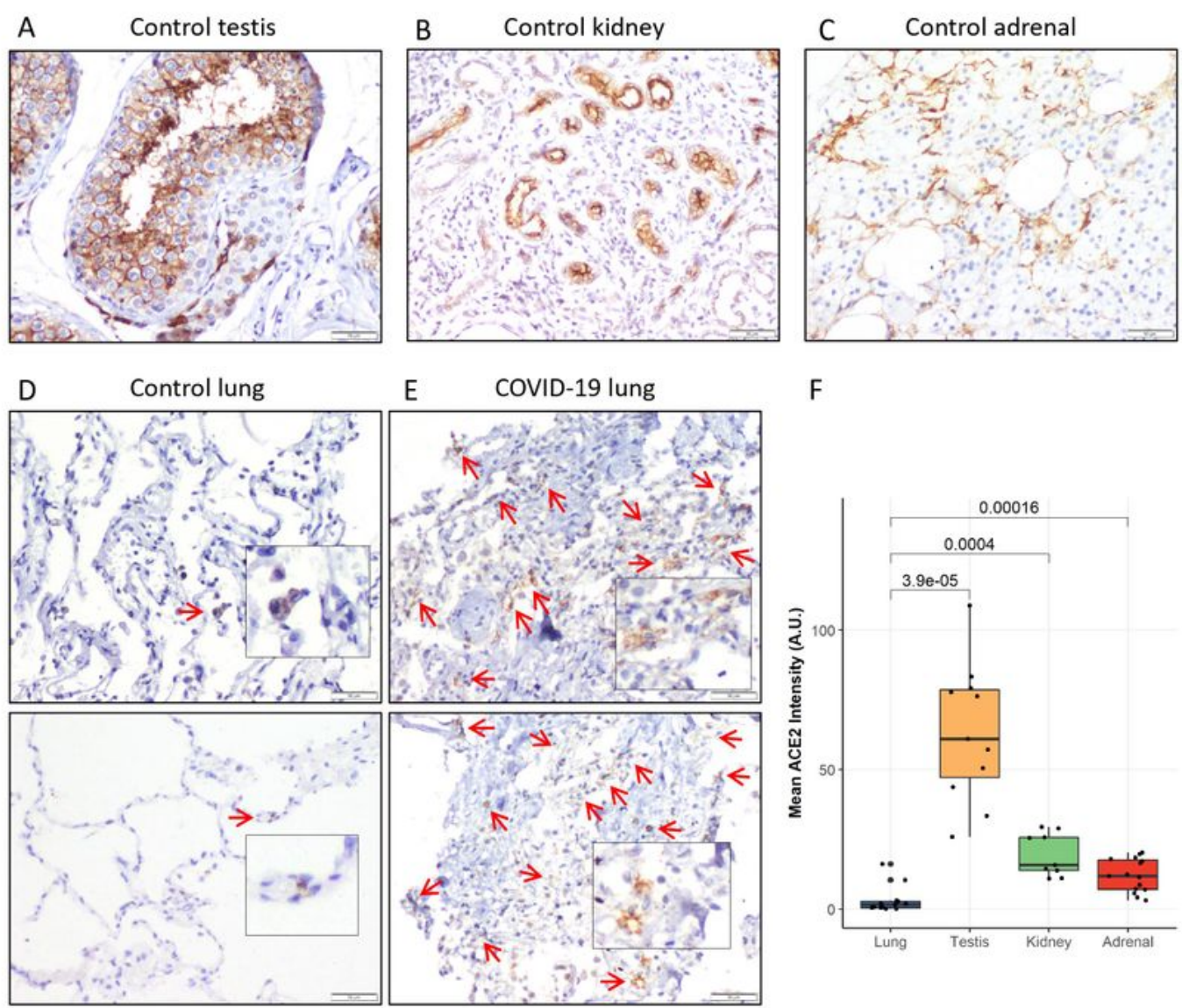

E COVID-19 lung

F
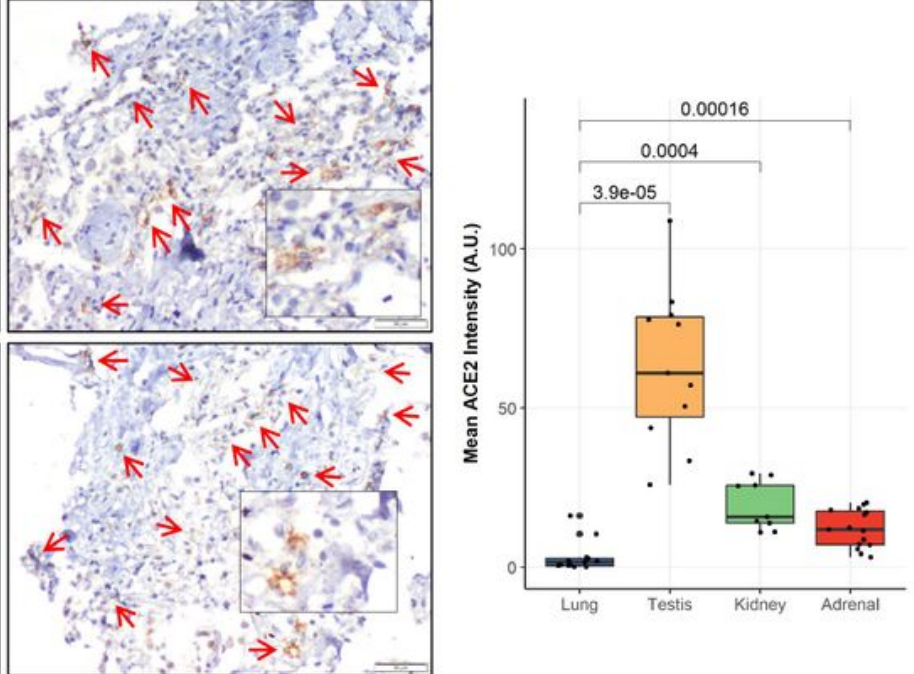

G

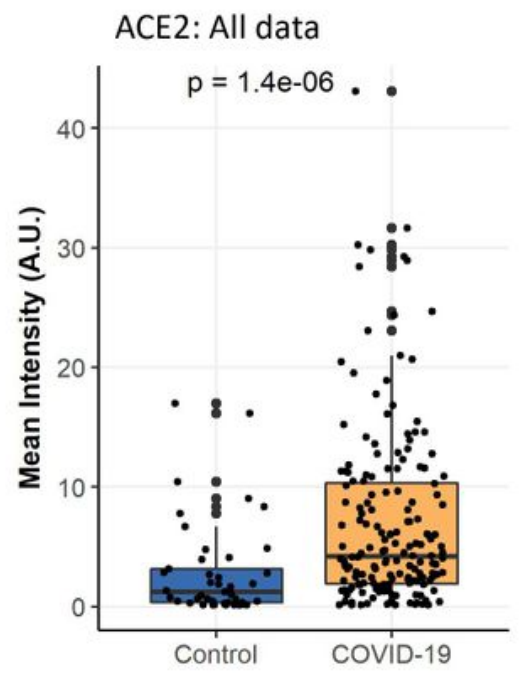

$\mathrm{H}$

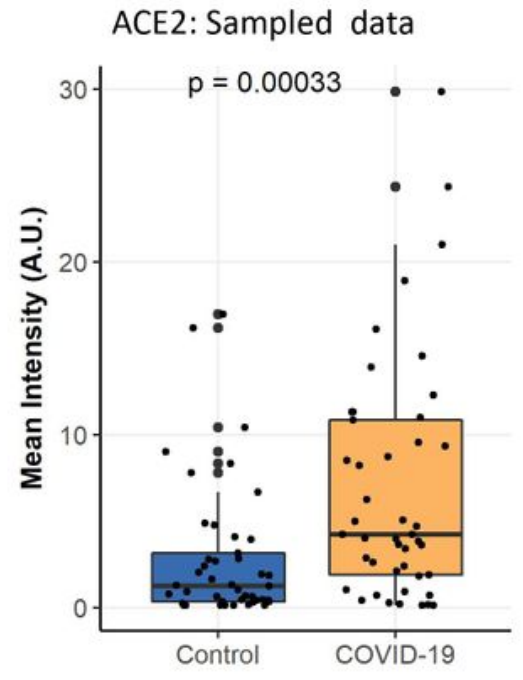

Figure 2 
Landscape of ACE2. Large patches of ACE2 positive staining in control tissue of (A) testis, (B) kidney, and (C) in adrenal. Representative images of (D) control and (E) COVID-19 infected lung sections showing increased ACE2 staining in COVID-19 cases (brown colour, arrows, and insets, D and E). (F) Quantitative analysis of ACE2 IHC intensity in various control tissues. The difference in the mean intensities was calculated using Wilcox test. Significance denoted by exact P-values ( $\mathrm{n}=13$ random images from 15 control lung tissue section, $n=11$ images from 2 control testis tissue section, $n=9$ images from 2 control kidney tissue section, $n=15$ images from 2 control adrenal tissue section). ( $G$ and $H$ ) Quantitative analysis of ACE2 IHC intensity in control and COVID-19 lung section $(G)$ without sampling analysis $(n=45$ images from 15 control lung tissue section and $n=175$ images from 44 COVID-19 lung tissue section) and $(H)$ with random sampling analysis ( $n=45$ images in each group). The difference in the mean intensities was calculated using the Wilcox test, and the exact P-value denoted significance. 

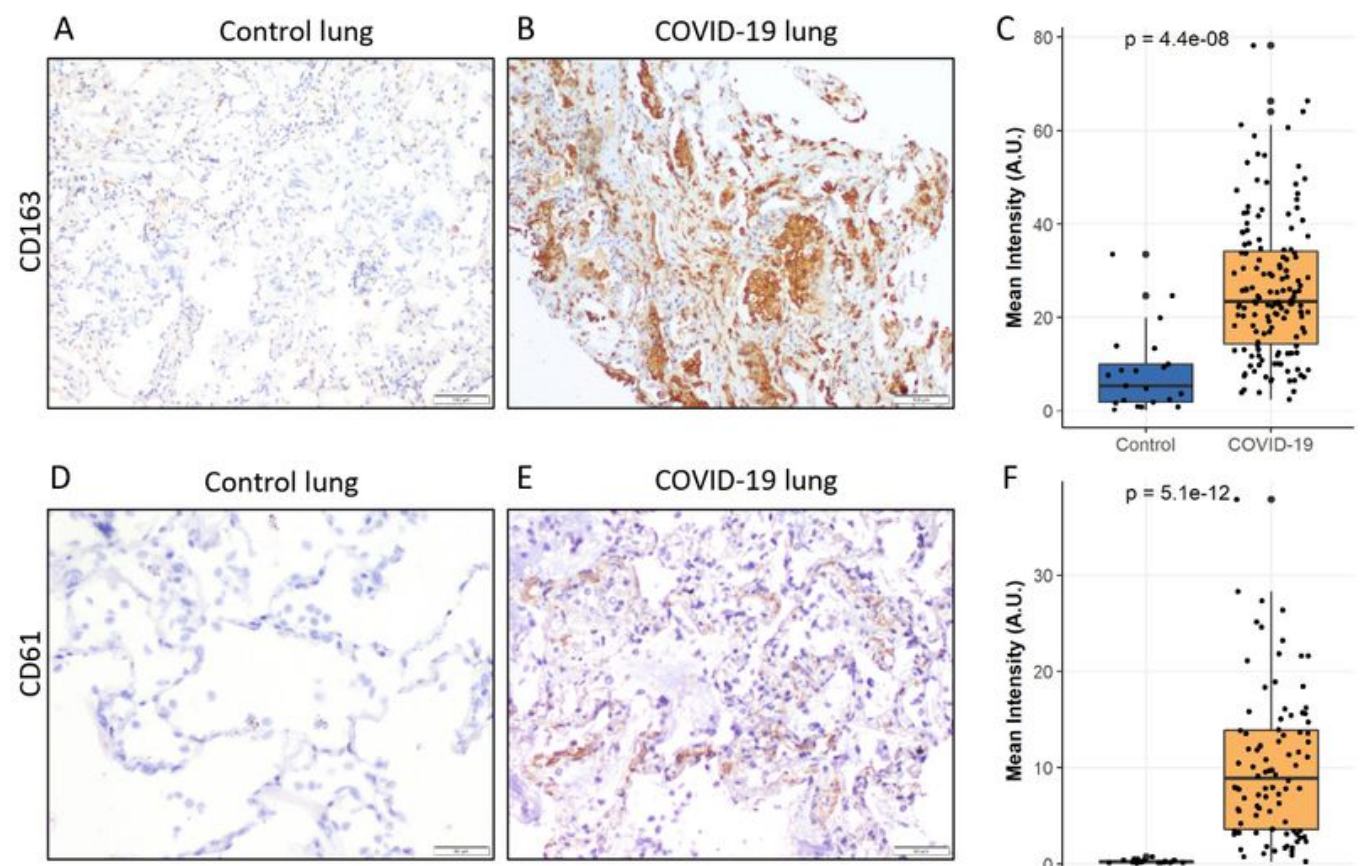

G
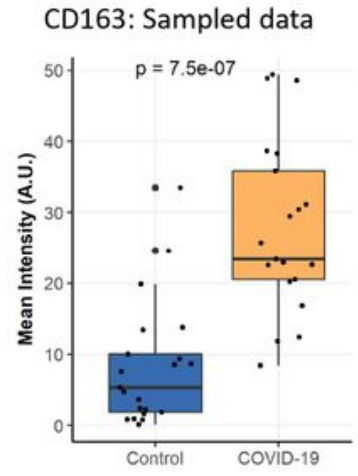

H CD61: Sampled data
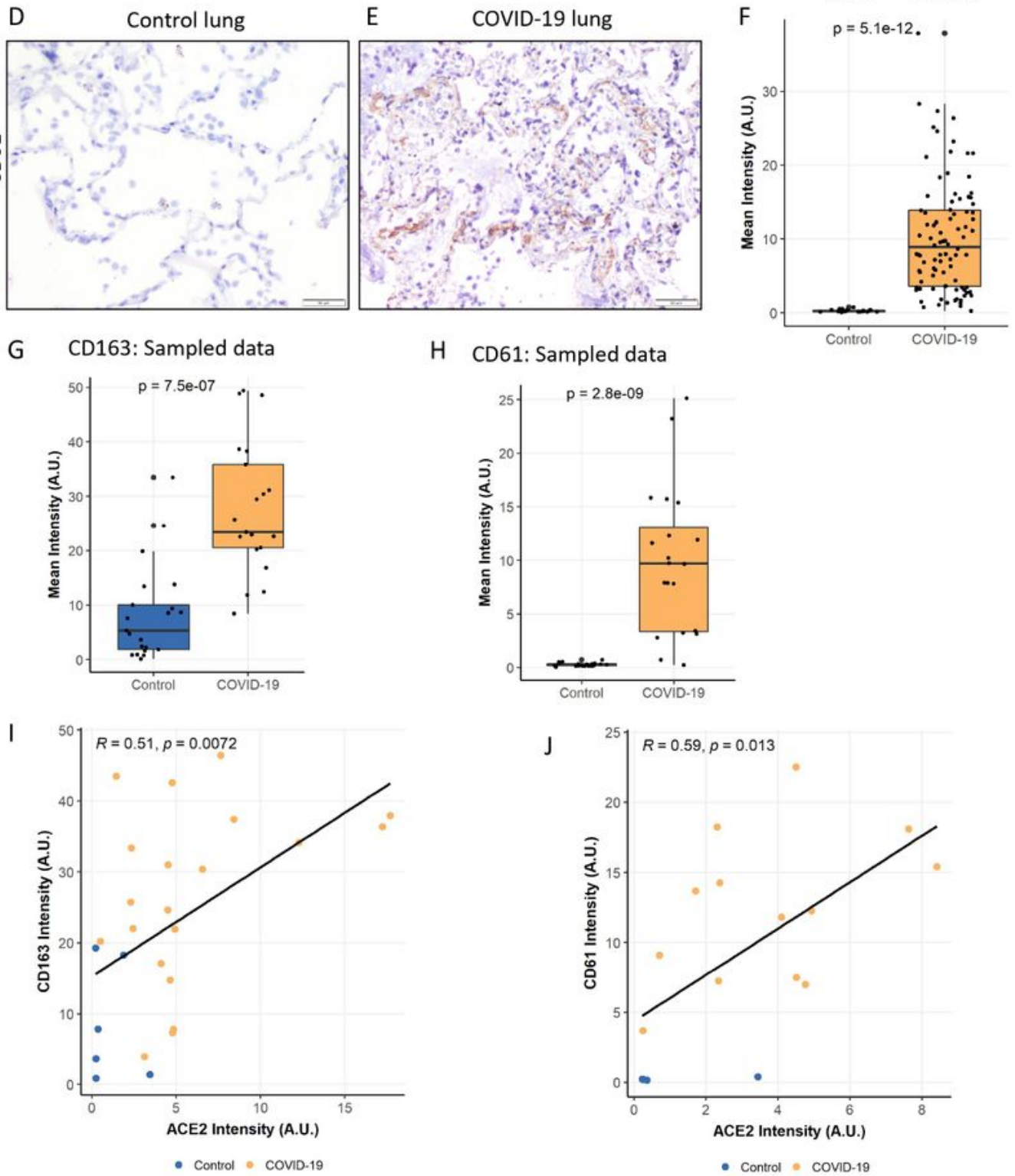

\section{Figure 3}

The landscape of CD163 and CD61. Representative images of (A) control and (B) COVID-19 infected lung sections showing increased CD163 staining in COVID-19 cases, with (C) mean Intensities of CD163 in control and COVID-19 samples. The difference in the mean intensities was calculated using Wilcox test Significance denoted by exact P-values ( $n=21$ images from 6 control lung tissue sections and $n=157$ images from 36 COVID-19 lung tissue sections). Detection of CD61 protein in representative sections of 
(D) control and (E) COVID-19 lung. (F) Mean intensity levels of CD61 staining in control and COVID-19 lungs. ( $n=20$ images from 6 control lung tissue section and $n=94$ images from 26 COVID-19 lung tissue section). ( $G$ and $H$ ) Intensities of CD163 ( $n=21$ images/group) and CD61 ( $n=20$ images/group) protein in control and COVID-19 lung section after random sampling analysis. The difference in the mean intensities was calculated using the Wilcox test. Significance denoted by exact P-values. (I and J) Correlation between ACE2-CD163 and ACE2-CD61 has been shown. A regression line was calculated using by fitting a linear model to the data. Label at the top shows correlation value $(R)$ and $P$-value for the linear fit.

\section{Supplementary Files}

This is a list of supplementary files associated with this preprint. Click to download.

- Supplementaryinformation.docx 